\title{
The Discrete-Time Bounded-Real Lemma in Digital Filtering
}

\author{
P. P. VAIDYANATHAN, MEMBER, IEEE
}

\begin{abstract}
The Lossless Bounded-Real lemma is developed in the discrete-time domain, based only on energy balance arguments. The results are used to prove a discrete-time version of the general Bounded-Real lemma, based on a matrix spectral-factorization result that permits a transfer matrix embedding process. Some applications of the results in digital filter theory are finally outlined.
\end{abstract}

\section{INTRODUCTION}

$\mathrm{I}$ $\mathrm{N}$ THE continuous-time world, the description of passivity properties of linear time-invariant systems in terms of minimal state-space descriptions is well known. The application of such results in system theory, network synthesis, and in stability analysis is also well established [1],[2]. For example, given a Lossless Positive Real (LPR) matrix $\boldsymbol{Z}(s)$, any minimal state-space description satisfies the LPR Lemma [1]. A generalization for lossy impedance matrices is the Positive Real Lemma or PR Lemma, which states the PR property in terms of the state-space matrices $(\boldsymbol{A}, \boldsymbol{B}, \boldsymbol{C}, \boldsymbol{D})$. Scattering domain versions of these descriptions are also known, and are contained in the BoundedReal Lemma (BR Lemma) and the Lossless Bounded-Real Lemma.

The proofs of the above lemma are known to be tedious, and an excellent survey can be found in [1]. A typical procedure is to establish the PR lemma, and then obtain the LPR lemma as a special case. The scattering domain versions are then obtained by translating the statements of the (Lossless) PR lemma into the scattering domain by simple wave-transformations, even though a direct development, not based on the PR counterpart, is possible. Discrete-time versions of these results are easily obtained by careful application of the bilinear transformation. For example, the discrete-time LPR lemma is developed in [2], based on this approach.

The purpose of this paper is to reconsider the discretetime BR lemma. We outline an independent proof of the lemma without invoking the PR counterpart or any related results in the continuous-time world. We begin by proving the discrete-time $L B R$ (DTLBR) lemma, based only on energy-balance arguments. We then extend this to the case of BR matrices (DTBR lemma) by invoking a classic result on spectral factorization [3]. Note that this approach is unlike the conventional approach, where the BR (or PR)

Manuscript received October 24,1984 . This work was supported in part by the National Science Foundation under Grant ECS 84-04245.

The author is with the Department of Electrical Engineering, California Institute of Technology, Pasadena, CA 91125. lemma is first established, with the LBR (or LPR) lemma following as a special case.

As an interesting application of the DT(L)BR lemma in the field of digital filtering, we revisit in Section IV certain well-known structures such as the Gray and Markel cascaded lattice structures, the Barnes and Fam minimumnorm structures, wave filters, and orthogonal digital filters, with the help of the DT $(L)$ BR lemma.

We trust that the developments are simple and elegant. Moreover, once the DTLBR and DTBR lemmas are established in this way, it leads to a complete set of proofs of the BR, LBR, PR, and LPR lemmas, both in the continuoustime and discrete-time domains. This is because of the well-known bilinear transformation [4] and the BR to PR transformations [1].

\section{Notations and Definitions}

In this paper, bold faced letters denote vectors and matrices. Superscript $t$ denotes matrix-transpose, whereas superscript dagger $\left({ }^{\dagger}\right)$ denotes transposed conjugate. The tilde accent is defined as follows: $\tilde{\boldsymbol{H}}(z)=\boldsymbol{H}^{t}\left(z^{-1}\right)$. The symbol $\boldsymbol{P}^{-t}$ stands for "inverse of the transpose of $\boldsymbol{P}$ ". The rotation $\boldsymbol{A} \leqslant \boldsymbol{B}$, where $\boldsymbol{A}$ and $\boldsymbol{B}$ are square matrices, is an abbreviation for " $\boldsymbol{A}-\boldsymbol{B}$ is negative semi-definite." The symbol $I$ stands for the identity matrix of appropriate dimension, whereas 0 denotes a null vector or null matrix.

A matrix, each of whose entries is a ratio of two polynomials with real coefficients, is termed a real rational matrix function. A real rational $p \times m$ transfer matrix $\boldsymbol{H}(z)$ is said to be bounded real (BR) if (a) each of its entries represents an asymptotically stable system, and in addition, (b) $\boldsymbol{H}^{\dagger}\left(e^{j \omega}\right) \boldsymbol{H}\left(e^{j \omega}\right) \leqslant \boldsymbol{I}$ for all $\omega$. Such a transfer matrix is said to be lossless bounded-real ( $L B R$ ) if equality holds in (b) for all $\omega$. An equivalent characterization of condition (b) is that for every (finite-energy) input sequence $u(n)$, the output sequence $y(n)$ of the system satisfies

$$
\sum_{0}^{\infty} \boldsymbol{y}^{\dagger}(n) \boldsymbol{y}(n) \leqslant \sum_{0}^{\infty} \boldsymbol{u}^{\dagger}(n) \boldsymbol{u}(n)
$$

where the initial state $\boldsymbol{x}(0)$ is assumed to be zero. Moreover, equality in the above equation for all sequences $\boldsymbol{u}(n)$ corresponds to equality in (b) for all $\omega$.

\section{Discrete-Time Version of the LBR Lemma}

Let $\boldsymbol{H}(z)$ be a $p \times m$ real rational-transfer matrix of an $m$-input $p$-output system. Let the real matrices $(\boldsymbol{A}, \boldsymbol{B}, \boldsymbol{C}, \boldsymbol{D})$ represent a minimal state-space description of the system, 
i.e.,

$$
\begin{aligned}
\boldsymbol{x}(n+1) & =\boldsymbol{A} \boldsymbol{x}(n)+\boldsymbol{B u}(n) \\
\boldsymbol{y}(n) & =\boldsymbol{C} \boldsymbol{x}(n)+\boldsymbol{D} \boldsymbol{u}(n)
\end{aligned}
$$

with $(\boldsymbol{A}, \boldsymbol{B})$ completely controllable and $\left(\boldsymbol{A}, \boldsymbol{C}^{t}\right)$ completely observable. Here $\boldsymbol{A}$ is an $N \times N$ matrix, where $N$ is the McMillan degree of $\boldsymbol{H}(z)$. The input-output relation is

$$
\boldsymbol{Y}(z)=\boldsymbol{H}(z) \boldsymbol{U}(z)
$$

where

$$
\boldsymbol{H}(z)=\boldsymbol{D}+\boldsymbol{C}(z \boldsymbol{I}-\boldsymbol{A})^{-1} \boldsymbol{B} .
$$

The discrete-time LBR (DTLBR) lemma can be stated as follows: $\boldsymbol{H}(z)$ is $L B R$ if and only if there exists a real symmetric positive definite matrix $\boldsymbol{P}$ such that

$$
\begin{aligned}
\boldsymbol{A}^{t} \boldsymbol{P A}+\boldsymbol{C}^{t} \boldsymbol{C} & =\boldsymbol{P} \\
\boldsymbol{B}^{t} \boldsymbol{P B}+\boldsymbol{D}^{t} D & =\boldsymbol{I} \\
\boldsymbol{A}^{t} \boldsymbol{P B}+\boldsymbol{C}^{t} \boldsymbol{D} & =\mathbf{0} .
\end{aligned}
$$

Proof of the DTLBR Lemma: We first show that if (5) holds, then $\boldsymbol{H}(z)$ is LBR, and then show the converse. Accordingly, assume first that (5) is true. In particular, consider (5a), with $\boldsymbol{P}=\boldsymbol{P}^{t}>0$. Because of minimality $\left(\boldsymbol{A}, \boldsymbol{C}^{t}\right.$ ) is completely observable, hence by Lyapunov lemma [1], (5a) implies that all the eigenvalues of $\boldsymbol{A}$ are strictly within the unit circle. Thus $\boldsymbol{H}(z)$ is asymptotically stable.

Next, since $\boldsymbol{P}=\boldsymbol{P}^{t}>0$, we can decompose it as $\boldsymbol{P}=$ $\boldsymbol{T}^{-t} \boldsymbol{T}^{-1}$, and rewrite (5) as

$$
\begin{aligned}
T^{t} A^{t} T^{-t} T^{-1} A T+T^{t} C^{t} C T=I \\
B^{t} T^{-t} T^{-1} B+D^{t} D=I \\
T^{t} A^{z} T^{-t} T^{-1} B+T^{t} C^{t} D=0
\end{aligned}
$$

where $\boldsymbol{T}$ is real, nonsingular. Thus an equivalent state-space description of the system $\boldsymbol{H}(z)$ is obtained as follows:

$$
\begin{array}{r}
\boldsymbol{x}_{1}(n+1)=\boldsymbol{A}_{1} \boldsymbol{x}_{1}(n)+\boldsymbol{B}_{1} \boldsymbol{u}(n) \\
\boldsymbol{y}(n)=\boldsymbol{C}_{1} \boldsymbol{x}_{1}(n)+\boldsymbol{D}_{1} \boldsymbol{u}(n)
\end{array}
$$

where

$$
A_{1}=T^{-1} A T \quad B_{1}=T^{-1} B \quad C_{1}=C T \quad D_{1}=D
$$

In view of (6), the matrices $\left(A_{1}, B_{1}, C_{1}, D_{1}\right)$ are such that the $(p+N) \times(m+N)$ matrix $\mathscr{R}$ defined as

$$
\mathscr{R} \triangleq\left[\begin{array}{ll}
A_{1} & B_{1} \\
C_{1} & D_{1}
\end{array}\right]
$$

is an "orthogonal" matrix, i.e., $\mathscr{R}^{t} \mathscr{R}=\boldsymbol{I}$. Accordingly, the equality:

$\boldsymbol{x}_{1}^{t}(n+1) \boldsymbol{x}_{1}(n+1)+\boldsymbol{y}^{t}(n) \boldsymbol{y}(n)$

$$
=\boldsymbol{x}_{1}^{t}(n) \boldsymbol{x}_{1}(n)+\boldsymbol{u}^{t}(n) \boldsymbol{u}(n)
$$

always holds, hence

$$
\begin{array}{r}
\sum_{n=0}^{M} \boldsymbol{y}^{t}(n) \boldsymbol{y}(n)=\sum_{n=0}^{M} \boldsymbol{u}^{t}(n) \boldsymbol{u}(n)+\boldsymbol{x}_{1}^{t}(0) \boldsymbol{x}_{1}(0) \\
-\boldsymbol{x}_{1}^{t}(M+1) \boldsymbol{x}_{1}(M+1)
\end{array}
$$

for every integer $M>0$. If we assume $\boldsymbol{u}(n)=0$ for $n>M$, then

$$
\text { i.e., } \quad \begin{aligned}
y(n) & =C_{1} x_{1}(n), \quad n>M \\
y^{t}(n) y(n) & =x_{1}^{t}(n) C_{1}^{t} C_{1} x_{1}(n) \\
& =x_{1}^{t}(n)\left[I-A_{1}^{t} A_{1}\right] x_{1}(n)
\end{aligned}
$$

by orthogonality of $\mathscr{R}$. Thus

$$
\begin{aligned}
\sum_{n=M+1}^{\infty} \boldsymbol{y}^{t}(n) \boldsymbol{y}(n) & \\
& =\sum_{n=M+1}^{\infty}\left[\boldsymbol{x}_{1}^{t}(n) \boldsymbol{x}_{1}(n)-\boldsymbol{x}_{1}^{t}(n+1) \boldsymbol{x}_{1}(n+1)\right] \\
& =\boldsymbol{x}_{1}^{t}(M+1) \boldsymbol{x}_{1}(M+1) .
\end{aligned}
$$

Equations (12) and (15) imply that

$$
\sum_{n=0}^{\infty} \boldsymbol{y}^{t}(n) \boldsymbol{y}(n)=\sum_{n=0}^{\infty} \boldsymbol{u}^{t}(n) \boldsymbol{u}(n)+\boldsymbol{x}_{1}^{t}(0) \boldsymbol{x}_{1}(0)
$$

for every finite energy input that is identically zero for $n>M$, where $M$ is any arbitrary finite integer. This implies that $\boldsymbol{H}(z)$ is indeed LBR.

We now proceed to establish the converse. For this, assuming that $\boldsymbol{H}(z)$ is LBR, let us obtain a state-space description $\left(A_{1}, B_{1}, C_{1}, D_{1}\right)$ such that $\mathscr{R}$ defined by $(10)$ is orthogonal. Let $(\boldsymbol{A}, \boldsymbol{B}, \boldsymbol{C}, \boldsymbol{D})$ represent some minimal realization of $\boldsymbol{H}(z)$. As $\boldsymbol{H}(z)$ is asymptotically stable, there exists a real matrix $\boldsymbol{P}=\boldsymbol{P}^{t}>0$ such that (5a) holds. Once again, decomposing $\boldsymbol{P}$ according to $\boldsymbol{P}=\boldsymbol{T}^{-t} \boldsymbol{T}^{-1}$, definc $\left(\boldsymbol{A}_{1}, \boldsymbol{B}_{1}, \boldsymbol{C}_{1}, \boldsymbol{D}_{1}\right)$ as in (9). The following equality then holds:

$$
A_{1}^{t} A_{1}+C_{1}^{t} C_{1}=I
$$

Now, by LBR property of $\boldsymbol{H}(z)$ we have the following equality:

$$
\sum_{n=0}^{\infty} \boldsymbol{y}^{t}(n) \boldsymbol{y}(n)=\sum_{n=0}^{\infty} \boldsymbol{u}^{t}(n) \boldsymbol{u}(n)
$$

for any finite-energy input, applied under zero initial-conditions. In particular, let $\boldsymbol{u}(n)$ be zero for $n>M$, where $M$ is any arbitrary finite positive integer. Then (13) holds, hence (15) follows, by using (17). Thus (18) can be rewritten as

$$
\sum_{n=0}^{M} \boldsymbol{y}^{t}(n) \boldsymbol{y}(n)=\sum_{n=0}^{M} \boldsymbol{u}^{t}(n) \boldsymbol{u}(n)-\boldsymbol{x}_{1}^{t}(M+1) \boldsymbol{x}_{1}(M+1)
$$

By repeating (19) with $M$ replaced by $M+1$, and then subtracting, we get

$$
\begin{aligned}
& {\left[x_{1}^{t}(M+1) y^{t}(M)\right]\left[\begin{array}{c}
x_{1}(M+1) \\
y(M)
\end{array}\right]} \\
& =\left[x_{1}^{t}(M) u^{t}(M)\right]\left[\begin{array}{c}
x_{1}(M) \\
u(M)
\end{array}\right]
\end{aligned}
$$

for any integer $M>0$. This implies that the matrix $\mathscr{R}$ defined by $(10)$ is orthogonal, which proves the desired result. 
Note that $\mathscr{R}$ defined by $(10)$ cannot satisfy $\mathscr{R} \mathscr{R}=\mathrm{I}$ unless $p \geqslant m$. Thus any $p \times m$ LBR transfer matrix $\boldsymbol{H}(z)$ must have $p \geqslant m$.

\section{Discrete-Time Version OF THE BR LemMA}

Let us now proceed to the more general case, where $\boldsymbol{H}(z)$ is not necessarily lossless, but is still BR. The discrete-time BR-lemma (DTBR lemma) can be stated as follows:

The DTBR Lemma: Let $\boldsymbol{H}(z)$ be a real rational $p \times m$ transfer matrix, and let the real matrices $(A, B, C, D)$ represent a minimal state-space realization as in (1),(2). Then $\boldsymbol{H}(z)$ is BR if and only if there exist real matrices $\boldsymbol{L}$, $\boldsymbol{W}$, and a real symmetric positive definite matrix $\boldsymbol{P}$, such that

$$
\begin{aligned}
A^{t} P A+C^{t} C+L^{t} L & =P \\
B^{t} P B+D^{t} D+W^{t} W & =I \\
A^{t} P B+C^{t} D+L^{t} W & =0 .
\end{aligned}
$$

Assuming that (21) holds, let us first show that $\boldsymbol{H}(z)$ is indeed BR. Thus (21) imply the existence of an equivalent minimal realization $\left(\boldsymbol{A}_{1}, \boldsymbol{B}_{1}, \boldsymbol{C}_{1}, \boldsymbol{D}_{1}\right)$ such that

$$
\begin{aligned}
A_{1}^{t} A_{1}+C_{1}^{t} C_{1}+L_{1}^{t} L_{1} & =I \\
B_{1}^{t} B_{1}+D_{1}^{t} D_{1}+W_{1}^{t} W_{1} & =\boldsymbol{I} \\
A_{1}^{t} B_{1}+C_{1}^{t} D_{1}+L_{1}^{t} W_{1} & =\mathbf{0}
\end{aligned}
$$

where $\left(\boldsymbol{A}_{1}, \boldsymbol{B}_{1}, \boldsymbol{C}_{1}, \boldsymbol{D}_{1}\right)$ are given by (9), and where $\boldsymbol{T}$ is a real matrix such that $\boldsymbol{P}=\boldsymbol{T}^{-t} \boldsymbol{T}^{-1}$. Moreover,

$$
\boldsymbol{L}_{1}=\boldsymbol{L} \boldsymbol{T} \quad \boldsymbol{W}_{1}=\boldsymbol{W}
$$

are real matrices. Clearly, the transfer matrix $\boldsymbol{G}(z)$ described by $\left(\boldsymbol{A}_{1}, \boldsymbol{B}_{1}, \boldsymbol{C}_{2}, \boldsymbol{D}_{2}\right)$ where

$$
C_{2}=\left(\begin{array}{l}
C_{1} \\
L_{1}
\end{array}\right) \quad D_{2}=\left(\begin{array}{l}
D_{1} \\
W_{1}
\end{array}\right)
$$

is LBR, by the DTLBR lemma. Moreover,

$$
H(z)=D_{1}+C_{1}\left(z I-A_{1}\right)^{-1} B_{1}
$$

whereas

$$
\boldsymbol{G}(z)=\left[\begin{array}{l}
D_{1}+C_{1}\left(z I-A_{1}\right)^{-1} B_{1} \\
W_{1}+L_{1}\left(z I-A_{1}\right)^{-1} B_{1}
\end{array}\right]=\left[\begin{array}{l}
H(z) \\
H_{1}(z)
\end{array}\right]
$$

Since $\boldsymbol{G}(z)$ is LBR, it immediately follows that

$$
\boldsymbol{H}^{\dagger}\left(e^{j \omega}\right) \boldsymbol{H}\left(e^{j \omega}\right)=\boldsymbol{I}-\boldsymbol{H}_{1}^{\dagger}\left(e^{j \omega}\right) \boldsymbol{H}_{1}\left(e^{j \omega}\right)
$$

hence $\boldsymbol{H}(\mathrm{z})$ is $\mathrm{BR}$.

Next consider the converse. Assuming that $\boldsymbol{H}(z)$ is indeed BR, let us consider an irreducible [5] right matrixfraction description (MFD) for $\boldsymbol{H}(z)$ :

$$
\boldsymbol{H}(z)=\mathscr{N}(z) \mathscr{D}^{-1}(z)
$$

Here $\mathscr{N}(z)$ and $\mathscr{D}(z)$ are a $p \times m$ and $m \times m$ polynomial matrices, respectively. Since $\boldsymbol{H}(z)$ is BR, the matrix polynomial defined by

$$
\tilde{\mathscr{D}}(z) \mathscr{D}(z)-\tilde{\mathscr{N}}(z) \mathscr{N}(z)
$$

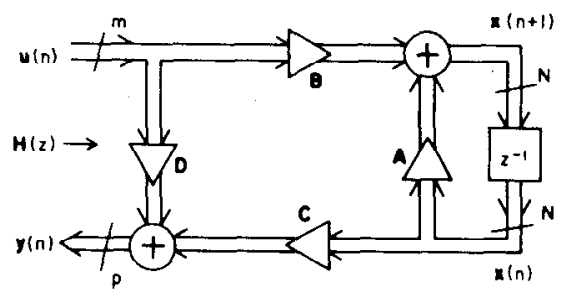

Fig. 1. The circuit-interpretation.

is positive semidefinite for $z=e^{j \omega}$. Thus based on thc classic result of Youla on matrix-factorization ([3, th. 2, corollary 2]), there exists an $r \times m$ matrix polynomial $\mathscr{K}(z)$ with real coefficients such that

$$
\tilde{\mathscr{K}}(z) \mathscr{K}(z)=\tilde{\mathscr{D}}(z) \mathscr{D}(z)-\tilde{\mathscr{N}}(z) \mathscr{N}(z)
$$

where $r$ is the normal rank of the matrix polynomial in (29). Let us define a new transfer matrix $\boldsymbol{G}(z)$ by the following right MFD:

$$
\boldsymbol{G}(z)=\left[\begin{array}{c}
\mathscr{N}(z) \\
\mathscr{K}(z)
\end{array}\right] \mathscr{D}^{-1}(z) .
$$

In other words, $\boldsymbol{H}(z)$ is embedded into $\boldsymbol{G}(z)$. Clearly, $\boldsymbol{G}(z)$ is asymptotically stable because $\boldsymbol{H}(z)$ is BR. Moreover, by construction of $\mathscr{K}(z), \boldsymbol{G}(z)$ is LBR. If $(\boldsymbol{A}, \boldsymbol{B}, \boldsymbol{C}, \boldsymbol{D})$ is a minimal realization of $\boldsymbol{H}(z)$, then $(\boldsymbol{A}, \boldsymbol{B}, \hat{\boldsymbol{C}}, \hat{\boldsymbol{D}})$ is a minimal realization of $\boldsymbol{G}(z)$ where

$$
\hat{C}=\left[\begin{array}{l}
C \\
L
\end{array}\right] \quad \hat{D}=\left[\begin{array}{l}
D \\
W
\end{array}\right]
$$

for some real matrices $L, W$. Since $G(z)$ is $L B R$, it is clear from the DTLBR lemma that the following equations hold:

$$
\begin{aligned}
\boldsymbol{A}^{t} \boldsymbol{P A}+\hat{\boldsymbol{C}}^{t} \hat{\boldsymbol{C}} & =\boldsymbol{P} \\
\boldsymbol{B}^{t} \boldsymbol{P B}+\hat{\boldsymbol{D}}^{t} \hat{\boldsymbol{D}} & =\boldsymbol{I} \\
\boldsymbol{A}^{t} \boldsymbol{P B}+\hat{\boldsymbol{C}}^{t} \hat{\boldsymbol{D}} & =\mathbf{0}
\end{aligned}
$$

for some real $\boldsymbol{P}=\boldsymbol{P}^{t}>0$. Thus (21) is immediately seen to hold. This then establishes the converse statement in the DTBR lemma.

\section{Circuit Interpretation}

For discrete-time systems, a very simple circuit-interpretation can be attached to the DT(L)BR lemma. Thus consider Fig. 1, which shows an implementation of a $p \times m$ transfer matrix $\boldsymbol{H}(z)$ with $N$ delays where $N$ is the McMillan degree of $\boldsymbol{H}(z)$. After all the delays have been extracted, we are left with a $(p+N)$-output $(m+N)$-input memoryless system, characterized by the (constant) transfer matrix.

$$
\mathscr{R}=\left[\begin{array}{ll}
\boldsymbol{A} & \boldsymbol{B} \\
\boldsymbol{C} & \boldsymbol{D}
\end{array}\right]
$$

The DTBR lemma says that $\boldsymbol{H}(z)$ is BR if and only if we can find a minimal implementation such that $\mathscr{R}$ is upper bounded as follows:

$$
\mathscr{R}^{t} \mathscr{R} \leqslant \boldsymbol{I} .
$$

Thus Fig. 1 would then represent a passive memoryless structure, constrained at $N$ terminals by (lossless) delay elements. The DTLBR lemma says that $\boldsymbol{H}(z)$ is LBR if 
and only if there exists a minimal implementation such that $\mathscr{R}$ is orthogonal, i.e., lossless. Thus Fig. 1 then represents a lossless memoryless structure, constrained at $N$ terminals by delay elements. A possible application of this interpretation is in the synthesis of low-sensitivity digital filter structures, based on the concept of structural passivity [6], [14]. It can be shown that structures based only on planar-rotation building blocks can be derived in this manner, but the details are beyond the scope of this paper. However, in the next section, some applications in the area of digital filtering are outlined.

\section{Applications in Digital Filtering}

In this section, we make use of the DT(L)BR lemma in order to revisit certain well-known results in the literature on digital filters.

\section{Limit-Cycle Free Digital Filters}

Given an arbitrary stable transfer function $H(z)$ of order $N$, assume without loss of generality that it is BR (see Section II). According to the DTBR lemma, we can therefore find a minimal state-space realization $(\boldsymbol{A}, \boldsymbol{B}, \boldsymbol{C}, d)$ such that

$$
A^{t} A+C_{1}^{t} C_{1}=I
$$

where $\boldsymbol{C}_{1}^{t}=\left(\begin{array}{ll}C^{t} & \boldsymbol{L}^{t}\end{array}\right)$ for some real $\boldsymbol{L}$. Under zero-input conditions, we then have

$$
\begin{aligned}
\boldsymbol{x}^{\dagger}(n+1) \boldsymbol{x}(n+1) & =\boldsymbol{x}^{\dagger}(n) \boldsymbol{A}^{t} \boldsymbol{A} \boldsymbol{x}(n) \\
& \leqslant \boldsymbol{x}^{\dagger}(n) \boldsymbol{x}(n)-\boldsymbol{x}^{\dagger}(n) C_{1}^{t} C_{1} \boldsymbol{x}(n) .
\end{aligned}
$$

Now by minimality of the given realization $\left[A, C^{t}\right]$ is observable, hence $\boldsymbol{C x}(n)$ cannot be zero for $N$ successive instants of time, unless $\boldsymbol{x}(n)$ is identically zero. In order to see this, let us assume the contrary, i.e.,

$$
\boldsymbol{C} \boldsymbol{x}(n)=\mathbf{0}, \quad n_{0} \leqslant n \leqslant n_{0}+N-1
$$

with $\boldsymbol{x}\left(n_{0}\right) \neq \mathbf{0}$. Thus

$$
\left[\begin{array}{c}
\boldsymbol{C} \\
\boldsymbol{C A} \\
\vdots \\
\boldsymbol{C} \boldsymbol{A}^{N-1}
\end{array}\right] \boldsymbol{x}\left(n_{0}\right)=\mathbf{0}, \quad \boldsymbol{x}\left(n_{0}\right) \neq \mathbf{0}
$$

which violates the complete-observability condition. Hence the quantity

$$
\boldsymbol{x}^{\dagger}(n) C_{1}^{\ell} C_{1} \boldsymbol{x}(n)
$$

cannot be zero for $N$ successive instants of time, hence from (37) the internal energy $\boldsymbol{x}^{\dagger}(n) \boldsymbol{x}(n)$, which is a nonincreasing function of $n$, has to strictly decrease at least once in a period of length $N$. In summary, we have the following lemma:

Lemma 4.1: Let $H(z)$ be $N$ th-order BR and let $(\boldsymbol{A}, \boldsymbol{B}, \boldsymbol{C}, d)$ be a minimal realization satisfying the DTBR lemma. Then, under zero input conditions, the internal energy $\boldsymbol{x}^{\dagger}(n) \boldsymbol{x}(n)$ is a nonincreasing sequence, and strictly decreases at least once in every $N$ units of time.

The lemma clearly holds also for LBR systems. Let us now consider a minimal state-space structure, as in Fig. 2,

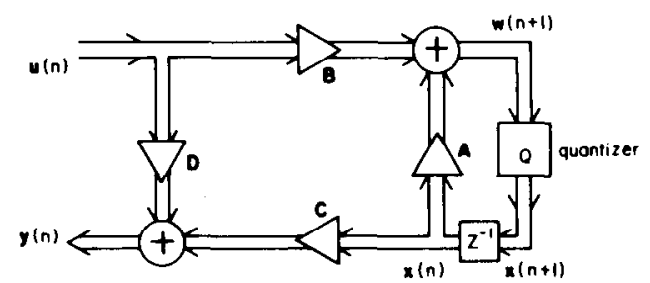

Fig. 2. The DTRR structure under quantization.

where signal-quantization is taken into account. Assume that $(\boldsymbol{A}, \boldsymbol{B}, \boldsymbol{C}, \boldsymbol{d})$ satisfies the DTBR lemma, and assume the quantizer to be "magnitude-truncation" type, i.e.,

$$
\left|x_{i}(n+1)\right|^{2} \leqslant\left|w_{i}(n+1)\right|^{2}
$$

for each $i$. Then, under zero-input conditions, the following inequalities hold:

$$
\begin{aligned}
& \boldsymbol{x}^{\dagger}(n+1) \boldsymbol{x}(n+1) \leqslant \boldsymbol{w}^{\dagger}(n+1) \boldsymbol{w}(n+1) \\
& \boldsymbol{w}^{\dagger}(n+1) \boldsymbol{w}(n+1) \leqslant \boldsymbol{x}^{\dagger}(n) \boldsymbol{x}(n) .
\end{aligned}
$$

If (40) is a strict inequality, then $\boldsymbol{x}^{\dagger}(n+1) \boldsymbol{x}(n+1)$ is smaller than $\boldsymbol{x}^{\dagger}(n) \boldsymbol{x}(n)$ by at least $\Delta^{2}$ where $\Delta$ is the smallest nonzero quantization error possible. On the other hand, if (40) is an equality for all $n>$ some $n_{0}$, then it implies $x_{i}^{2}(n+1)=w_{i}^{2}(n+1)$, i.e., $\boldsymbol{w}(n+1)=\boldsymbol{x}(n+1)$, hence the closed-loop system becomes "linear." Then by Lemma 4.1, $\boldsymbol{x}^{\dagger}(n) \boldsymbol{x}(n)$ should undergo a "strict decrease" during a period of length $N$. This in turn implies that equality in (40) cannot hold for all $n>n_{0}$ unless $x(n)$ itself has decayed to zero and remains zero. In conclusion, the structure of Fig. 2 cannot sustain zero-input limit cycles, and over an interval of length $N$ there is a decrease of internal energy by at least an amount $\Delta^{2}$, until the internal energy becomes zero.

The normalized Gray and Markel lattice structures [7] and the Barnes and Fam minimum-norm structure [9] for digital filters are known for their ability to suppress limit cycles. Let us next take a closer look at these well-known structures with the help of the DTBR lemma.

\section{Relation to Gray and Markel Lattice Structures}

The relation between lattice filters and system-theoretic concepts is well known. For example, see [19] and references therein. In this section, we revisit these relations in the light of the DT(L)BR lemma.

Consider a scalar (i.e., single-input single-output) stable all-pass digital filter transfer function:

$$
H(z)=\frac{b_{N}+b_{N-1} z^{-1}+\cdots+z^{-N}}{1+b_{1} z^{-1}+\cdots+b_{N} z^{-N}} .
$$

Clearly, this is an LBR function; hence, according to the LBR lemma, it can be implemented as in Fig. 1, where the orthogonal matrix $\mathscr{R}$ is now

$$
\mathscr{R}=\left[\begin{array}{ll}
\boldsymbol{A} & \boldsymbol{B} \\
\boldsymbol{C} & d
\end{array}\right]
$$

$d$ being a scalar, $\boldsymbol{C}$ a row vector, $\boldsymbol{B}$ a column vector, and $\boldsymbol{A}$ an $N \times N$ matrix. As an example, consider a second- 


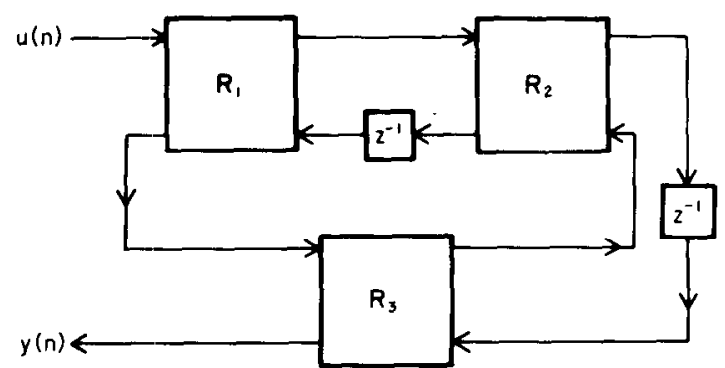

Fig. 3. Second-order all-pass filter, based on three rotations (each $\boldsymbol{R}_{k}$ is a $2 \times 2$ orthogonal transfer matrix).

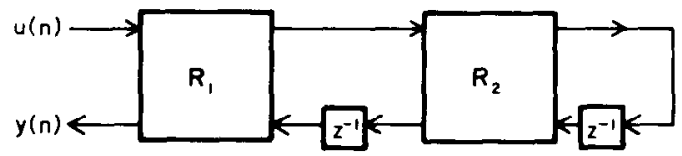

Fig. 4. The normalized Gray and Markel lattice structure for a secondorder all-pass filter.
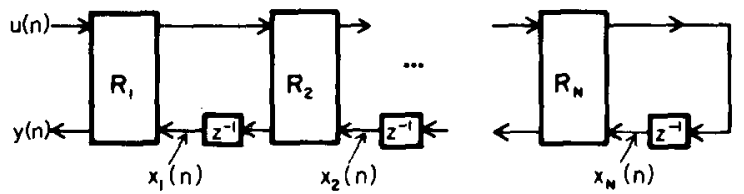

Fig. 5. The normalized cascaded lattice for $N$ th-order all pass (each $\boldsymbol{R}_{k}$ is a $2 \times 2$ orthogonal transfer matrix). where $\boldsymbol{C}=\left[\begin{array}{ll}c_{11} & c_{12}\end{array}\right]$. As $\boldsymbol{T}$ is orthogonal, the equivalent realization satisfies the DTLBR lemma, i.e.,

$$
\mathscr{R}_{1} \triangleq\left[\begin{array}{ll}
A_{1} & \boldsymbol{B}_{1} \\
\boldsymbol{C}_{1} & d
\end{array}\right]
$$

continues to be orthogonal, and has the form:

$$
\mathscr{R}_{1}=\left[\begin{array}{ccc}
-\cos \alpha \cos \theta & \sin \alpha & \cos \alpha \sin \theta \\
-\sin \alpha \cos \theta & -\cos \alpha & \sin \alpha \sin \theta \\
\sin \theta & 0 & \cos \theta
\end{array}\right]
$$

This can be implemented as in Fig. 4, requiring only 2 planar rotations, and leads to the normalized Gray and Markel lattice structure [7], which is well known for several excellent properties under finite-word length constraints. Notice that Fig. 3 reduces to Fig. 4 when $b=0$.

In the general case of an $N$ th order scalar allpass function, the normalized Gray and Markel lattice has the form shown in Fig. 5, and the $\mathscr{R}$ matrix is $(N+1) \times(N+1)$, having the form:

$$
\mathscr{R}=\left[\begin{array}{ll}
\boldsymbol{A} & \boldsymbol{B} \\
\boldsymbol{C} & d
\end{array}\right]=\left[\begin{array}{cccccc|c}
a_{11} & a_{12} & 0 & 0 & \cdots & 0 & b_{1} \\
a_{21} & a_{22} & a_{23} & 0 & \cdots & 0 & b_{2} \\
\vdots & & & & & & \vdots \\
a_{N-1,1} & a_{N-1,2} & \cdots & \cdots & \cdots & a_{N-1, N} & b_{N-1} \\
a_{N, 1} & a_{N, 2} & \cdots & \cdots & \cdots & a_{N, N} & b_{N} \\
\hline c_{1} & 0 & 0 & \cdots & \cdots & 0 & d
\end{array}\right] .
$$

order all-pass function. Then $\mathscr{R}$ is a $3 \times 3$ orthogonal matrix and can therefore be implemented in terms of 3 planar rotations:

$$
\begin{aligned}
& \mathscr{R}=\left[\begin{array}{ccc}
\cos a & \sin a & 0 \\
-\sin a & \cos a & 0 \\
0 & 0 & 1
\end{array}\right]\left[\begin{array}{ccc}
\cos b & 0 & -\sin b \\
0 & 1 & 0 \\
\sin b & 0 & \cos b
\end{array}\right] \\
& \cdot\left[\begin{array}{ccc}
1 & 0 & 0 \\
0 & \cos c & \sin c \\
0 & -\sin c & \cos c
\end{array}\right] .
\end{aligned}
$$

The implementation is, therefore, as in Fig. 3, and requires three "planar rotation" operators. It is, however, possible to apply a similarity transformation $\boldsymbol{T}$ to $(\boldsymbol{A}, \boldsymbol{B}, \boldsymbol{C}, d)$ to get an equivalent realization $\left(\boldsymbol{A}_{1}, \boldsymbol{B}_{1}, \boldsymbol{C}_{1}, d\right)$ where $\boldsymbol{C}_{1}$ has the form

$$
C_{1}=[\alpha, 0] .
$$

In order to do this $T$ can be picked as

$$
\boldsymbol{T}=\frac{\left[\begin{array}{cc}
c_{11} & c_{12} \\
c_{12} & -c_{11}
\end{array}\right]}{\sqrt{c_{11}^{2}+c_{12}^{2}}}
$$

It is shown in ([8, Appendix B]) that the above matrix is orthogonal. In other words, the structure of Fig. 5 satisfies DTLBR lemma equations. For convenience, let us define a matrix $\mathscr{P}$ as

$$
\mathscr{S}=\left[\begin{array}{ll}
d & C \\
B & A
\end{array}\right]
$$

Clearly $\mathscr{S}$ is orthogonal if and only if $\mathscr{R}$ is orthogonal. Thus the matrix $\mathscr{S}$ has the following structure:

$$
\mathscr{S}=\left[\begin{array}{cccccc}
s_{11} & s_{12} & 0 & \cdots & \cdots & 0 \\
s_{21} & s_{22} & s_{23} & 0 & \cdots & 0 \\
\vdots & & \ddots & & & \vdots \\
\vdots & & & \ddots & & \vdots \\
\vdots & & & & \ddots & \vdots \\
s_{N 1} & s_{N 2} & \cdots & \cdots & \cdots & s_{N, N+1} \\
s_{N+1,1} & s_{N+1,2} & \cdots & \cdots & \cdots & s_{N+1, N+1}
\end{array}\right]
$$

So, given an arbitrary state-space realization $\left(A_{1}, B_{1}, C_{1}, d\right)$ (for the all-pass function) that satisfies the DTLBR lemma, the normalized lattice is obtained by converting the orthogonal matrix

$$
\mathscr{S}_{1}=\left[\begin{array}{cc}
d & C_{1} \\
\boldsymbol{B}_{1} & \boldsymbol{A}_{1}
\end{array}\right]
$$


into the form of (50), by means of an orthogonal similarity transformation.

An incidental outcome of the above discussion is the following linear-algebra result: Given an arbitrary $(N+1)$ $\times(N+1)$ orthogonal matrix $\mathscr{S}_{1}$, it is always possible to find an orthogonal transformation $T$ such that

$$
\left[\begin{array}{cc}
1 & 0 \\
0 & T^{t}
\end{array}\right] \mathscr{S}_{1}\left[\begin{array}{cc}
1 & 0 \\
0 & T
\end{array}\right]=\mathscr{S}
$$

where $\mathscr{S}$ has the for in (50). Note that $\mathscr{P}$ is characterized by $N$ angles.

\section{Relation to the Barnes and Fam Minimal-Norm Digital Filters}

Barnes and Fam [9] have advanced a general theory for minimal-norm structures for digital filters, of which the well-known coupled-form [10] is a special case. Basically, if a stable digital filter transfer function has distincl poles, then a state-space realization can be obtained such that the $\boldsymbol{A}$ matrix is normal, i.e., $\boldsymbol{A}^{t} \boldsymbol{A}=\boldsymbol{A A}^{t}$. For such realizations, the $l_{2}$ norm of $A$ is strictly bounded by 1 :

$$
\sup _{\boldsymbol{y} \neq 0} \frac{\boldsymbol{y}^{t} A^{t} \boldsymbol{A y}}{\boldsymbol{y}^{t} \boldsymbol{y}}=\|\boldsymbol{A}\|_{2}^{2}<1
$$

i.e., according to our notations,

$$
\boldsymbol{A}^{t} \boldsymbol{A}<\boldsymbol{I} \text {. }
$$

Such structures, with normal $\boldsymbol{A}$ matrices are called minimum-norm structures. For a digital filter transfer function with distinct poles, one can always obtain structures of this kind [9]. The matrix $\boldsymbol{A}$ satisfying (54) is clearly a (strictly) $\mathrm{BR}$ matrix.

Notice, however, that any arbitrary stable (and BR) digital transfer function can always be realized as in Fig. 1, so that (36) holds. Even though this does not exactly imply (54), this does imply

$$
A^{t} \boldsymbol{A} \leqslant \boldsymbol{I} .
$$

According to our earlier discussions in this section, this is still sufficient to suppress limit cycles, as long as the realization is minimal (in particular, completely observable). In other words, even for non-minimum-norm realizations, limit cycles can be suppressed in this way.

\section{Relation to Orthogonal Digital Filters}

An excellent family of digital filter structures is the class of orthogonal filters, pioneered by DeWilde et al. [11], and studied by a number of other researchers [12]-[14]. These structures are known for several desirable properties such as modularity, low passband sensitivity, freedom from limit cycles, automatic internal-signal normalization, and so on.

We wish to point out here, one simple way to obtain an orthogonal implementation of a digital filter transfer matrix, based on the proof of the DTBR lemma of Section III. Recall that in Section III, while proving the converse in the DTBR lemma, we started with an arbitrary real rational $p \times m$ BR transfer matrix $\boldsymbol{H}(z)$ and embedded it into a $(p+r) \times m$ LBR transfer matrix $\boldsymbol{G}(z)$. The LBR transfer matrix $\boldsymbol{G}(z)$ has a state-space representation $(\boldsymbol{A}, \boldsymbol{B}, \hat{\boldsymbol{C}}, \hat{\boldsymbol{D}})$

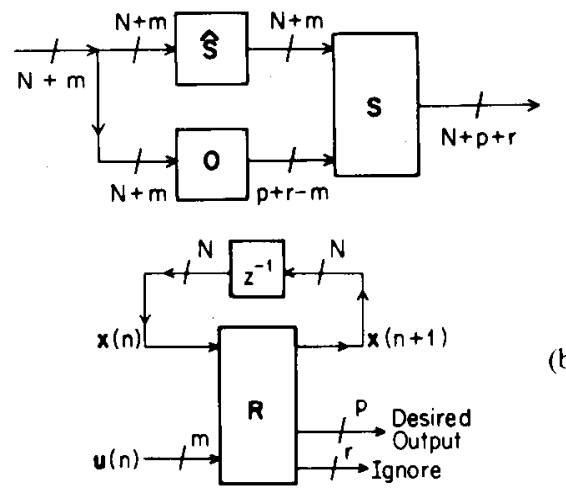

(b)

Fig. 6. (a) Implementation of the matrix $\mathscr{R}$. (b) Orthogonal implementation of $\boldsymbol{H}(z)$

satisfying (33). Recall also that, since $\boldsymbol{G}(z)$ is LBR, we must have $p+r \geqslant m$.

Let us now perform a similarity transformation of this state-space description as follows:

$$
\begin{aligned}
& \boldsymbol{A}_{0}=\boldsymbol{T}^{-1} \boldsymbol{A} \boldsymbol{T}, \\
& \boldsymbol{B}_{0}=\boldsymbol{T}^{-1} \boldsymbol{B}, \\
& \boldsymbol{C}_{0}=\hat{\boldsymbol{C}} \boldsymbol{T}, \\
& \boldsymbol{D}_{0}=\hat{\boldsymbol{D}}
\end{aligned}
$$

where $\boldsymbol{T}$ is defined so that $\boldsymbol{P}=\boldsymbol{T}^{-t} \boldsymbol{T}^{-1}$ (i.e., $\boldsymbol{T}^{-1}$ is a square root of the positive definite matrix $\boldsymbol{P}$ ). It is then clear that the description $\left(\boldsymbol{A}_{0}, \boldsymbol{B}_{0}, \boldsymbol{C}_{0}, \boldsymbol{D}_{0}\right)$ is such that the $(N+p+r) \times(N+m)$ matrix

$$
\mathscr{R}=\left[\begin{array}{ll}
\boldsymbol{A}_{0} & \boldsymbol{B}_{0} \\
\boldsymbol{C}_{0} & \boldsymbol{D}_{0}
\end{array}\right]
$$

is orthogonal. Now, simply by implementing the LBR transfer matrix $G(z)$, and ignoring the $r$ outputs corresponding to $\mathscr{K}(z)$ in (31), we can obtain an implementation of the BR transfer matrix $\boldsymbol{H}(z)$. In particular, if $\boldsymbol{G}(z)$ is implemented in state-space form $\left(\boldsymbol{A}_{0}, \boldsymbol{B}_{0}, \boldsymbol{C}_{0}, \boldsymbol{D}_{0}\right)$, then we have an orthogonal implementation of $\boldsymbol{H}(z)$. The $(N+$ $p+r) \times(N+m)$ matrix $\mathscr{R}$ which satisfies $\mathscr{R} \mathscr{R}^{t}=\boldsymbol{I}$ clearly has full column rank $N+m$, and can be rewritten as

$$
\mathscr{R}=\mathscr{S}\left[\begin{array}{l}
\hat{\mathscr{S}} \\
\mathbf{0}
\end{array}\right]
$$

where $\mathscr{S}$ is $(N+p+r) \times(N+p+r), \hat{\mathscr{S}}$ is $(N+m) \times(N$ $+m)$ and

$$
S^{\prime} S=I, \quad \hat{S}^{\prime} \hat{S}=I
$$

The square orthogonal matrices $S$ and $\hat{S}$ can be implemented as products of matrices representing planar rotations. For example,

$$
\mathscr{S}=\mathscr{S}_{1} \mathscr{S}_{2} \cdots \mathscr{S}_{M} \quad \hat{\mathscr{S}}=\hat{\mathscr{S}}_{1} \hat{\mathscr{S}}_{2} \ldots \hat{\mathscr{S}}_{L}
$$

where $\mathscr{S}_{1}$ might be of the form

$$
\mathscr{S}_{1}=\left[\begin{array}{cccccc}
1 & 0 & 0 & 0 & 0 & 0 \\
0 & 1 & 0 & 0 & 0 & 0 \\
0 & 0 & \cos \theta_{1} & 0 & \sin \theta_{1} & 0 \\
0 & 0 & 0 & 1 & 0 & 0 \\
0 & 0 & \sin \theta_{1} & 0 & -\cos \theta_{1} & 0 \\
0 & 0 & 0 & 0 & 0 & 1
\end{array}\right] .
$$


Methods for decomposing orthogonal matrices into products as in (60) are well known [15]. Fig. 6 demonstrates the implementation. Note that, this is meant only to be a demonstration, and computationally more efficient implementations can be found in [11], involving considerably less number of planar rotations.

\section{Relation to Wave-Digital Filters}

Fettweis has established, through a number of publications [16]-[18], the crucial role of passivity in the implementation of digital filters under finite wordlength constraints. In some recent contributions, [6],[8],[14], we show how the "LBR-approach" to low-sensitivity digital filter design is related to the wave digital filters. In particular, we invoke the DTLBR lemma in [14] for establishing one instance of such a relation. It is clear, therefore, that several interesting properties of the wave digital filters under finite-wordlength constraints can be interpreted in terms of the results of this paper.

\section{ACKNOWLEDGMENT}

The author wishes to thank Dr. B. D. O. Anderson, of the Australian National University, for helpful discussions.

\section{REFERENCES}

[1] B. D. O Anderson and S. Vongpanitlerd, Network Analysis and Synthesis. Englewood Cliffs, NJ: Prentice-Hall, 1973.

[2] L. Hitz and B. D. O. Anderson, "Discrete positive-real functions and their application to system stability," Proc. Inst. Elect. Eng., pp. 153-155, Jan. 1969

[3] D. C. Youla, "On the factorization of rational matrices," IEEE Trans. Inform. Theory, vol. IT-7, pp. 172-189, July 1961.

[4] A. Antoniou, Digital Filters, Analysis and Design, New York: McGraw-Hill, 1979.

[5] C. A. Desoer and J. D. Schulman, "Zeros and poles of matrix transfer functions and their dynamical interpretation," IEEE Trans. Circuits Syst., vol. CAS-21, pp. 3-7, Jan. 1974.
[6] P. P. Vaidyanathan and S. K. Mitra, "Low passband sensitivity digital filters: A generalized viewpoint and synthesis procedures," Proc. IEEE, vol. 72 , pp. 404-423, Apr. 1984.

[7] A. H. Gray, Jr. and J. D. Markel, "A normalized digital filter structure," IEEE Trans. Acoust., Speech, Signal Processing, vol. ASSP-23, pp. 268-277, June 1975.

[8] P. P. Vaidyanathan and S. K. Mitra, "Passivity properties of low sensitivity digital filter structures," IEEE Trans. Circuits Syst., vol CAS-32, pp. 217-224, Mar. 1985.

[9] C. W. Barnes and A. T. Fam, "Minimum norm recursive digital filters that are free of overflow limit cycles," IEEE Trans. Circuits Syst., vol. CAS-24, pp. 569-574, October 1977.

[10] A. V. Oppenheim and R. W. Schafer, Digital Signal Processing. Englewood Cliffs, NJ: Prentice-Hall, 1975.

[11] P. DeWilde and E. Deprettere, "Orthogonal cascade rcalization of real multiport digital filters," Int. J. Circuit Theory Appl., vol. 8, pp. 245-277, 1980.

[12] D. Henrot and C. T. Mullis, "A modular and orthogonal digital filter structure for parallel processing," in IEEE Int. on Acoustics, Speech, and Signal Processing, pp. 623-626, Apr. 1983.

[13] S. K. Rao and T. Kailath, "Orthogonal digital filters for VLSI implementation," IEEE Trans. Circuits Syst., vol. CAS-31, pp 933-945, Nov. 1984

[14] P. P. Vaidyanathan "A unified approach to orthogonal digital filters and wave digital filters, based on LBR two-pair extraction," IEEE Trans. Circuits Syst., vol. CAS-32, pp. 673-686, July 1985

[15] F. D. Murnaghan, The Unitary and Rotation Groups. Washington, DC: Spartan Books, 1962.

[16] A. Fettweis, "Digital filter structures related to classical filter networks," Arch. Elek. Ubetrangung., vol. 25, pp. 79-89, Feb. 1971.

[17] A. Fettweis, "Pseudopassivity, sensitivity, and stability of wave digital filters," IEEE Trans. Circuit Theory, vol. 19, pp. 668-673, Nov. 1972.

[18] A. Fellweis and K. Meerkotter, "Suppression of parasitic oscillations in wave filters," IEEE Trans. Circuits Syst., vol. CAS-22, pp. 239-246, Mar. 1975.

[19] B. Friedlander, "Lattice filters for adaptive processing." Proc. IEEE, vol. 70, pp. 829-867, Aug. 1982.

\section{W}

P. P. Vaidyanathan (S'80-M'83), for a photograph and biography please see page 224 of the March 1985 issues of this Transactions. 